EGU2020-10177

https://doi.org/10.5194/egusphere-egu2020-10177

EGU General Assembly 2020

(c) Author(s) 2020. This work is distributed under

the Creative Commons Attribution 4.0 License.

\title{
Volcanic hazard assessment and vulnerability analysis at San Miguel Volcano, El Salvador.
}

\author{
Diana Jimenez ${ }^{1}$, Laura Becerril ${ }^{2}$, Stefania Bartolini ${ }^{2}$, and Joan Martí ${ }^{2}$ \\ ${ }^{1}$ Gerardo Barrios University, Research and Innovation, El Salvador (djimenez@ugb.edu.sv) \\ ${ }^{2}$ Institute of Earth Sciences Jaume Almera, ICTJA-CSIC, Lluis Sole i Sabaris s/n, 08028 Barcelona, Spain
}

Despite being one of the most active volcanoes in El Salvador, San Miguel is surrounded by important population nuclei and infrastructures. We used existing historical records of past eruptive activity, available geological knowledge and monitoring data gathered over the past fifteen years to conduct the first comprehensive, long-term hazard assessment of this volcano, aimed at helping to reduce the potential risk it poses. We applied probabilistic methods (QVAST and HASSET) specifically designed for volcanic hazard assessment to conduct two hazard analyses, one with a forecasting time window of two years using information on volcanic activity over the past 430 years (historical period), and another with a forecasting window of six months, with information from the past 16 years (monitoring period). Using the information from this hazard assessment, we simulated: (1) the five most likely scenarios (ashfall scenarios, short-medium extent, and VEI 1-2); (2) other probable scenarios related to lava flows, based on the historical record of the volcano; (3) other possible scenarios related to PDCs with similar characteristics to those that occurred during its geological history; and (4) the most hazardous scenario (ashfall, lava flow, PDC) that has been identified from its geological record. Finally, we construct a qualitative integrated volcanic hazard map through the combination of the simulated scenarios. Finally, we developed an exposure analysis of the San Miguel volcano area by considering population distribution, land use, private houses, official buildings (hospitals, schools, etc.), and communication infrastructure, for the different hazard scenarios. In the particular case of private houses and official buildings, we estimated a Vulnerability Index for the hazardous areas, applying the Physical Vulnerability Methodology based on the characterization of the type of construction materials of walls and roofs. This approach identifies the elements at risk according to each potential hazard, thus providing the authorities with a comprehensive tool to better understand the problem and to define emergency plans to minimize risk.

This research has been partially funded by Grants I-COOPA20161 (CSIC) and EU (DG ECHO) Project EVE n. 826292 